\title{
Optimization and improvement of Halbach cylinder design
}

\author{
Bjørk, Rasmus; Bahl, Christian Robert Haffenden; Smith, Anders; Pryds, Nini
}

Published in:

Journal of Applied Physics

Link to article, DOI:

$10.1063 / 1.2952537$

Publication date:

2008

Document Version

Publisher's PDF, also known as Version of record

Link back to DTU Orbit

Citation (APA):

Bjørk, R., Bahl, C. R. H., Smith, A., \& Pryds, N. (2008). Optimization and improvement of Halbach cylinder design. Journal of Applied Physics, 104(1), 013910. https://doi.org/10.1063/1.2952537

\section{General rights}

Copyright and moral rights for the publications made accessible in the public portal are retained by the authors and/or other copyright owners and it is a condition of accessing publications that users recognise and abide by the legal requirements associated with these rights.

- Users may download and print one copy of any publication from the public portal for the purpose of private study or research.

- You may not further distribute the material or use it for any profit-making activity or commercial gain

- You may freely distribute the URL identifying the publication in the public portal

If you believe that this document breaches copyright please contact us providing details, and we will remove access to the work immediately and investigate your claim 


\title{
Optimization and improvement of Halbach cylinder design
}

\author{
R. Bjørk, ${ }^{a)}$ C. R. H. Bahl, A. Smith, and N. Pryds \\ Department of Fuel Cells and Solid State Chemistry, National Laboratory for Sustainable Energy, Technical \\ University of Denmark, Frederiksborgvej 399, DK-4000 Roskilde, Denmark
}

(Received 6 December 2007; accepted 7 May 2008; published online 9 July 2008)

\begin{abstract}
In this paper we describe the results of a parameter survey of a 16 segmented Halbach cylinder in three dimensions in which the parameters internal radius, $r_{\mathrm{in}}$, external radius, $r_{\mathrm{ex}}$, and length, $L$, have been varied. Optimal values of $r_{\mathrm{ex}}$ and $L$ were found for a Halbach cylinder with the least possible volume of magnets with a given mean flux density in the cylinder bore. The volume of the cylinder bore could also be significantly increased by only slightly increasing the volume of the magnets, for a fixed mean flux density. Placing additional blocks of magnets on the end faces of the Halbach cylinder also improved the mean flux density in the cylinder bore, especially so for short Halbach cylinders with large $r_{\text {ex }}$. Moreover, magnetic cooling as an application for Halbach cylinders was considered. A magnetic cooling quality parameter, $\Lambda_{\text {cool }}$, was introduced and results showed that this parameter was optimal for long Halbach cylinders with small $r_{\mathrm{ex}}$. Using the previously mentioned additional blocks of magnets can improve the parameter by as much as $15 \%$ as well as improve the homogeneity of the field in the cylinder bore. (C) 2008 American Institute of Physics.
\end{abstract}

[DOI: 10.1063/1.2952537]

\section{INTRODUCTION}

Configurations of permanent magnets that produce a strong homogeneous field in a confined region of space and a very weak field elsewhere are useful in many applications such as particle accelerators, ${ }^{1}$ nuclear magnetic resonance (NMR) apparatus, ${ }^{2}$ or magnetic cooling applications. ${ }^{3}$

The design known as a Halbach cylinder is especially good at producing this type of magnetic field. A Halbach cylinder is a long cylinder made of a magnetic material with a bore along the cylinder symmetry axis. The Halbach cylinder can be characterized by three parameters: the internal and external radii, $r_{\text {in }}$ and $r_{\mathrm{ex}}$, respectively, and the length, $L$. The magnetic material around the bore is magnetized such that the direction of magnetization at any point is at an angle

$$
\eta=2 \theta
$$

from the vertical axis. ${ }^{4,5}$ This arrangement means that a uniform field will be created across the bore in the vertical direction without creating, in the ideal case, any stray field outside the cylinder. Figure 1 shows a drawing of a Halbach cylinder.

It is well known that the flux density inside the bore of an infinitely long Halbach cylinder is ${ }^{5}$

$$
B=B_{r} \ln \left(\frac{r_{\mathrm{ex}}}{r_{\mathrm{in}}}\right),
$$

where $B_{r}$ is the remanent flux density of the magnetic material.

Halbach cylinders have previously been investigated in detail in two dimensions, but there exist only a few investigations of Halbach cylinders in three dimensions, where the effect of the finite length of the Halbach cylinder has been studied. In one example ${ }^{6}$ the reduction in flux density due to

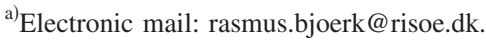

a finite length Halbach cylinder was investigated, but the field was only calculated for a single fixed length. An analytical formula for the magnetic flux of a Halbach cylinder of any given length was derived, however this formula is extremely complicated, making it impractical for direct application. In another publication ${ }^{7}$ the effects of a finite length Halbach cylinder were also explored, as well as the effect of dividing the Halbach cylinder into a number of segments, each with its own direction of magnetization. However, both investigations were only performed for one specific Halbach cylinder of a fixed length.

In this paper the three dimensional Halbach cylinder will be investigated in greater detail, and the flux density will be computed for a multitude of different configurations and not only a single specific case.

First, the effect of dividing the Halbach cylinder into segments each with their own direction of magnetization will

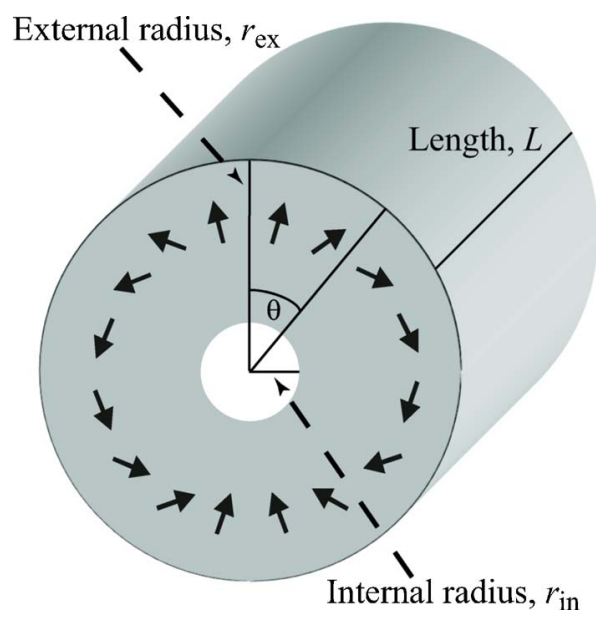

FIG. 1. (Color online) A sketch of a Halbach cylinder showing the internal radius, $r_{\text {in }}$, external radius, $r_{\mathrm{ex}}$, and length, $L$. Also shown as arrows is the direction of the remanent magnetization of the magnetic material. 
be investigated. To measure only the effect of segmentation the calculations are performed in two dimensions, so that any effects from a finite length Halbach cylinder are avoided. We then assume that the effect of segmentation in two dimensions is similar in three dimensions.

Thereafter the Halbach cylinder will be investigated in three dimensions, with focus on how to build a Halbach cylinder with a certain mean flux density using a minimum of magnetic material, i.e., find the configuration of $r_{\text {in }}, r_{\mathrm{ex}}$, and $L$ that generates the strongest flux density for the minimum amount of magnetic material.

Finally, it will be investigated if the magnetic flux density can be improved by placing additional blocks of permanent magnets on the end faces of the Halbach cylinder.

The results of this investigation of Halbach cylinder design are useful in many different fields, e.g., magnetic cooling $^{3}$ or tabletop NMR. ${ }^{8}$ These applications typically require a flux density of around 1-3 T, and this is also the range of flux density that we will concern ourselves with in this paper.

All numerical work in this paper was done using the commercially available finite element multiphysics program, Comsol Multiphysics. ${ }^{9}$ The Comsol Multiphysics code has previously been validated through a number of NAFEMS (National Agency for Finite Element Methods and Standards) benchmark studies. ${ }^{10}$

The equation solved in the following simulations is the magnetic vector potential equation,

$$
\nabla \times\left(\mu_{0}^{-1} \mu_{r}^{-1}\left(\nabla \times \mathbf{A}-\mathbf{B}_{r}\right)\right)=0,
$$

where $\mathbf{A}$ is the magnetic vector potential, $\mathbf{B}_{r}$ is the remanent flux density, $\mu_{0}$ is the permeability of free space, and $\mu_{r}$ is the relative permeability assumed to be isotropic.

The solver used to solve this equation on the simulation mesh is Pardiso, which is a parallel sparse direct linear solver. $^{11,12}$

Boundary conditions are chosen such that the boundaries of the computational volume, which is many times larger than the Halbach cylinder, are magnetically insulating, while all other (internal) boundaries are continuous.

\section{SEGMENTED HALBACH CYLINDER}

An infinitely long Halbach cylinder is equivalent to a two dimensional situation, so it fulfills Eq. (2) if the direction of magnetization varies continuously through the magnetic material as prescribed by Eq. (1). This continuous variation of the direction of magnetization is often not attainable in real-world assemblies, and therefore the Halbach cylinder is often made up of segments, each of which has a direction of magnetization equal to the direction of magnetization of a continuous Halbach cylinder at the center of the segment.

A Halbach cylinder consisting of $n$ such segments will have its flux density reduced to 5

$$
B(n)=B(\infty) \frac{\sin (2 \pi / n)}{2 \pi / n},
$$

where $B(\infty)$ is the flux density given by Eq. (2), i.e., with a continuous magnetization.

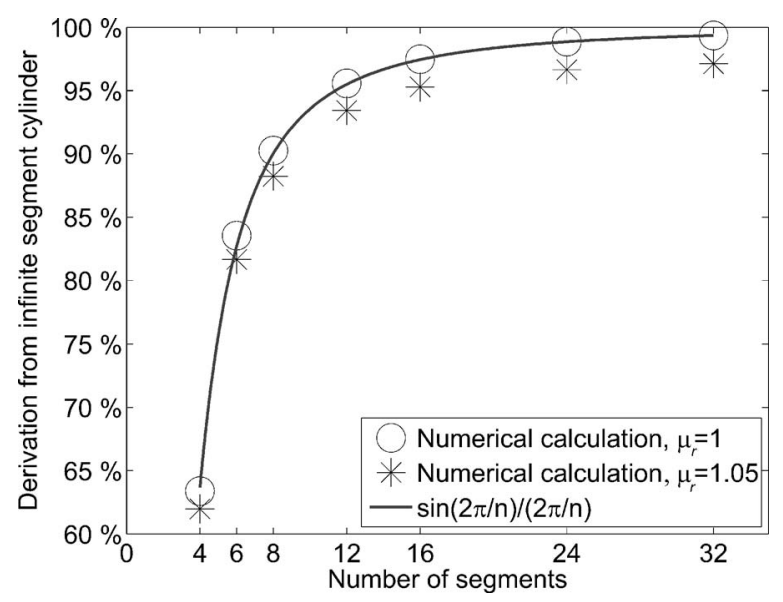

FIG. 2. Dividing a Halbach cylinder into $n$ segments makes the flux density differ from that of a perfect Halbach cylinder. The deviation is given by Eq. (4) for "perfect magnets," $\mu_{r}=1$, while magnets with $\mu_{r}=1.05$ deviate more from the ideal Halbach cylinder.

We have analyzed the consequence of this segmentation of the Halbach cylinder by computing the mean value of the magnetic flux density inside the Halbach cylinder bore for a Halbach cylinder consisting of 4, 6, 8, 12, 16, 24, and 32 segments. The calculations were performed both for a Halbach cylinder consisting of "perfect" magnets, i.e., with a relative permeability, $\mu_{r}$, of 1 , and magnets where actual material properties were taken into account by increasing $\mu_{r}$ to 1.05 . The magnetic material was assumed to have a remanent flux density of $1.4 \mathrm{~T}$, equal to standard grade N48 neodymium-iron-boron $(\mathrm{NdFeB})$ magnets. ${ }^{13}$ This value for the remanent flux density will be used in the remainder of this paper.

The results of the computations together with Eq. (4) are shown in Fig. 2. Here it is seen that Eq. (4) describes the numerical data with $\mu_{r}=1$ extremely well. It is also seen that choosing a small number of segments severely limits the flux density.

Based on the results shown in Fig. 2 we choose, in the calculations and computations presented in the following sections, to use a 16 segmented Halbach cylinder with $\mu_{r}$ $=1.05$. This configuration obtains $95 \%$ of the flux density of a perfect Halbach cylinder and is realizable in real-world assemblies.

Having determined the configuration to be used in the following simulations we now proceed to investigate if there exist optimal dimensions for a Halbach cylinder design. For this three dimensional simulation must be used in order to study how the loss of flux density through the ends of the cylinder bore varies with $r_{\mathrm{ex}}$ and $L$.

\section{HALBACH CYLINDER 3D STUDY}

A parameter study of Halbach cylinder configurations has been performed by varying the parameters $L, r_{\mathrm{ex}}$, and $r_{\text {in }}$ as given in Table I. In each of the $90 \times 90 \times 3$ configurations the mean flux density of the magnetic field inside the cylinder bore has been computed. The results are shown as a contour plot of the mean flux density as a function of $L$ and $r_{\text {ex }}$ in Fig. 3 for $r_{\text {in }}=20 \mathrm{~mm}$. 
TABLE I. The variation of the Halbach parameters. In total there are 90 $\times 90 \times 3$ different configurations.

\begin{tabular}{lccc}
\hline \hline & From $(\mathrm{mm})$ & To $(\mathrm{mm})$ & Step size $(\mathrm{mm})$ \\
\hline$L$ & 41 & 130 & 1 \\
$r_{\mathrm{ex}}$ & 22 & 200 & 2 \\
$r_{\text {in }}$ & 10 & 30 & 10 \\
\hline
\end{tabular}

Figure 3 shows that the configuration producing the strongest mean flux density is the configuration with the largest $r_{\mathrm{ex}}$ and $L$. This is in agreement with Eq. (2) and the fact that for a long Halbach cylinder the loss of flux through the ends of the cylinder will be relatively smaller than for a short cylinder.

It is not sufficient to characterize a design only by the value of the mean flux density. It should be considered that increasing the length of the Halbach cylinder increases the volume of the bore, thus allowing a larger sample to be placed inside the Halbach cylinder bore. On the other hand, increasing the external radius does not affect the volume of the bore. Consequently, a better way of characterizing each Halbach cylinder configuration is by the volume of its magnets and the volume of the bore, and then calculating contour plots with lines of equal mean flux density. These are shown in Figs. 4-6 for the three different values of $r_{\text {in }}$. In Figs. 4-6 the volume of the bore scales directly with the length of the Halbach cylinder because the internal radius is kept constant in each figure.

Looking at, e.g., Fig. 5 it can be seen that for a mean flux density of $1.6 \mathrm{~T}$ a Halbach cylinder can be constructed with an $\sim 50 \%$ increase in magnetic material but an $\sim 250 \%$ larger volume of the bore compared to the design using the least amount of magnetic material.

It is possible to attain this substantial increase in the volume of the bore because the latter configuration is a very long Halbach cylinder with a small external radius, while the configuration with the smallest volume of the magnets is a

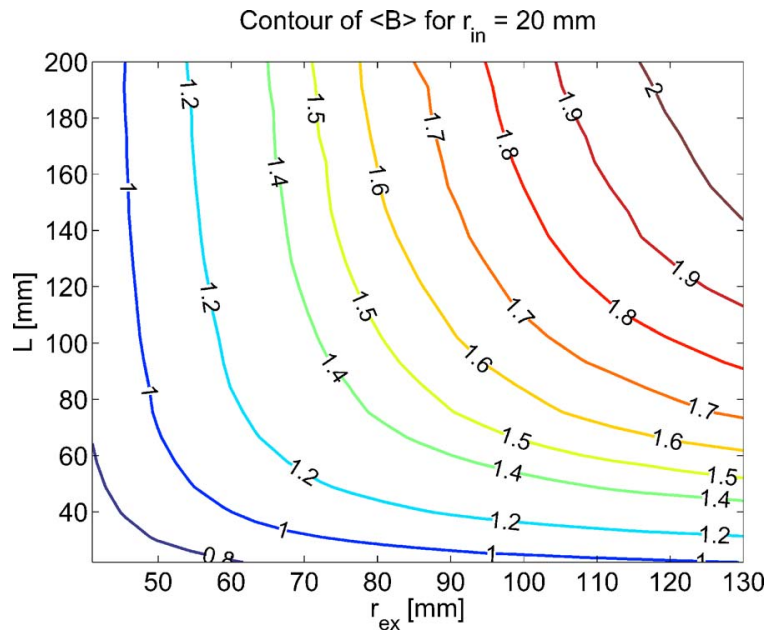

FIG. 3. (Color online) Contours of the mean flux density for the Halbach cylinders with $r_{\text {in }}=20 \mathrm{~mm}$. Each contour is labeled by its mean flux density. As is expected the maximum flux density is obtained by maximizing both $r_{\mathrm{ex}}$ and $L$.

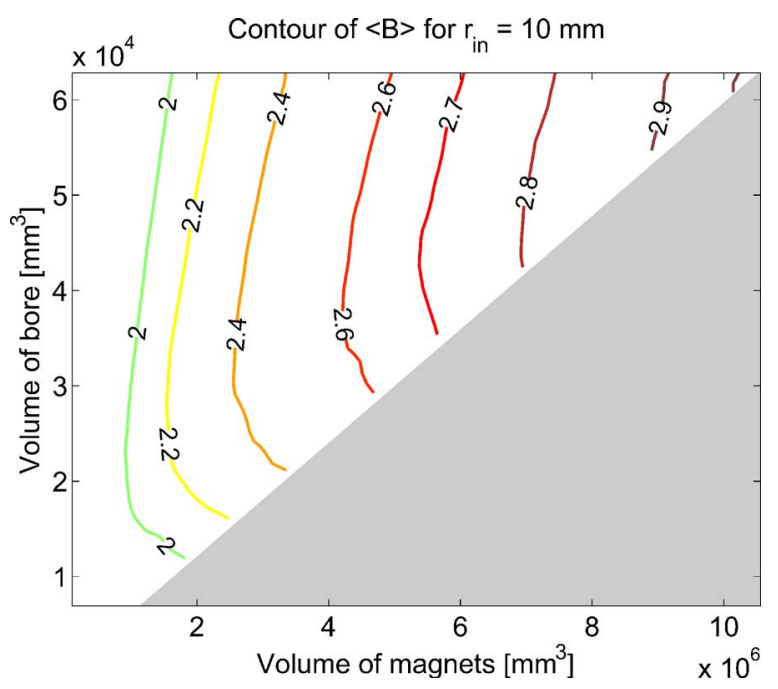

FIG. 4. (Color online) Contours of the mean flux density as a function of the volume of magnets used and the volume of the cylinder bore for $r_{\text {in }}$ $=10 \mathrm{~mm}$. It is seen that the volume of the bore can be significantly increased by slightly increasing the volume of the magnets. Note that the range is not the same on the two axes. A look-up table is necessary such that each data point (these are not shown) is uniquely tied to a specific Halbach cylinder, i.e., a given $r_{\text {in }}, r_{\mathrm{ex}}$, and $L$.

short Halbach cylinder with a large external radius. In these two configurations the shape of the bore is different, but the mean flux density.

In Fig. 7 the total volume of the magnetic material is shown as a function of the mean flux density in the bore for $r_{\text {in }}=20 \mathrm{~mm}$. In this plot there are $90 \times 90$ data points. Two data series where $r_{\mathrm{ex}}$ has been fixed and $L$ has been varied are highlighted on the plot. (One could also have chosen to vary $r_{\mathrm{ex}}$ and kept $L$ fixed. The curves look the same.) Here one can see that as $L$ is increased, the mean flux density is increased as well. At some point each data series becomes the rightwards edge of the "feather," and then the increase in the volume of the magnets with mean flux density becomes too steep and the data points move upwards, leaving the edge of the feather.

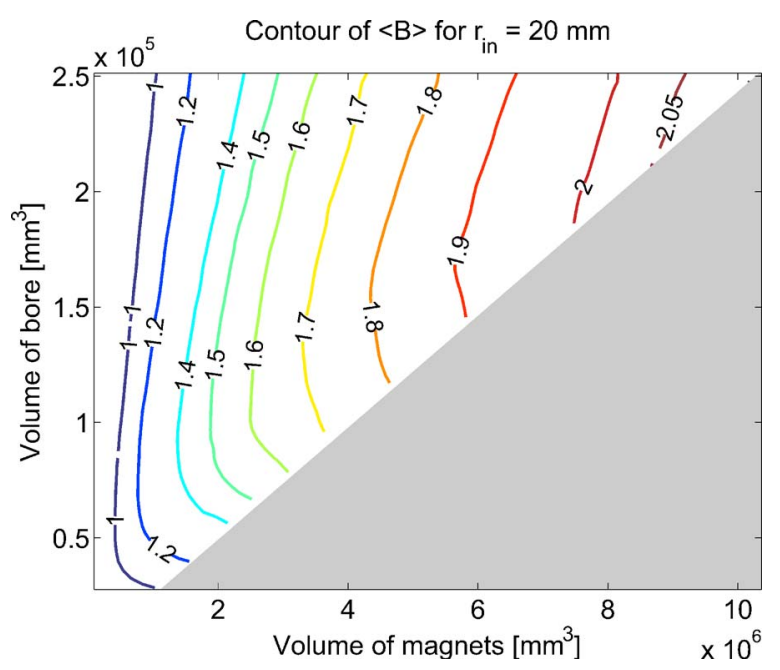

FIG. 5. (Color online) Contours of the mean flux density as a function of the volume of magnets used and the volume of the cylinder bore for $r_{\text {in }}$ $=20 \mathrm{~mm}$. The conclusion of Fig. 4 applies here as well. 


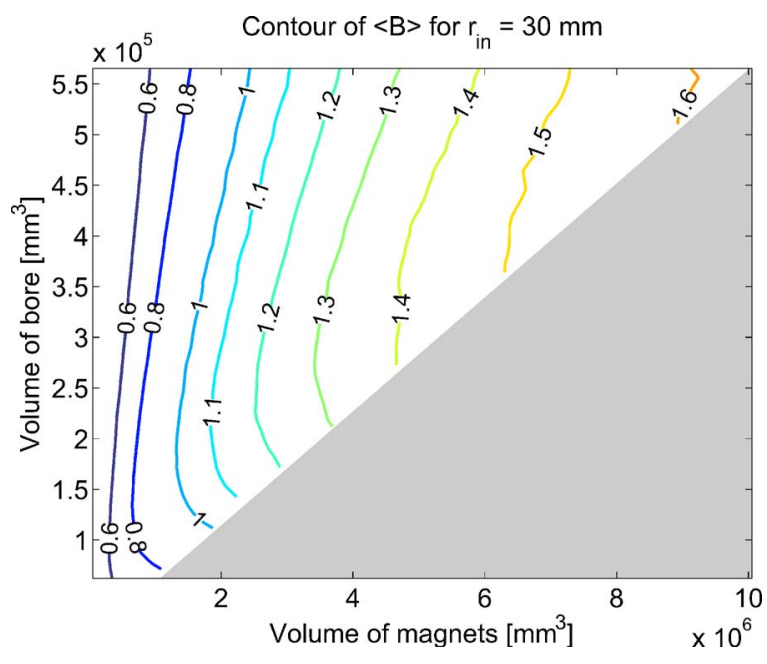

FIG. 6. (Color online) Contours of the mean flux density as a function of the volume of magnets used and the volume of the cylinder bore for $r_{\text {in }}$ $=30 \mathrm{~mm}$. The conclusion of Fig. 4 applies here as well.

The reason for this behavior is that the data series start with a short Halbach cylinder. This configuration loses a lot of flux through the ends of the cylinder and so as the length is increased the average flux density increases quite rapidly. When a certain length of the Halbach cylinder is reached there is not as much to be gained by increasing the length of the cylinder further and so the average flux density only increases slowly as the volume of the magnets is increased.

As this is the case for all data series where the length of the Halbach cylinder is gradually increased, it is possible to characterize the rightwards edge of the feather as the optimal configuration, i.e., the configuration with the smallest volume of the magnets at a given mean flux density.

In Fig. 8 the values of $r_{\mathrm{ex}}$ and $L$ are plotted as functions of the mean flux density for the optimal points. Thus one can directly use this figure to find the external radius and length

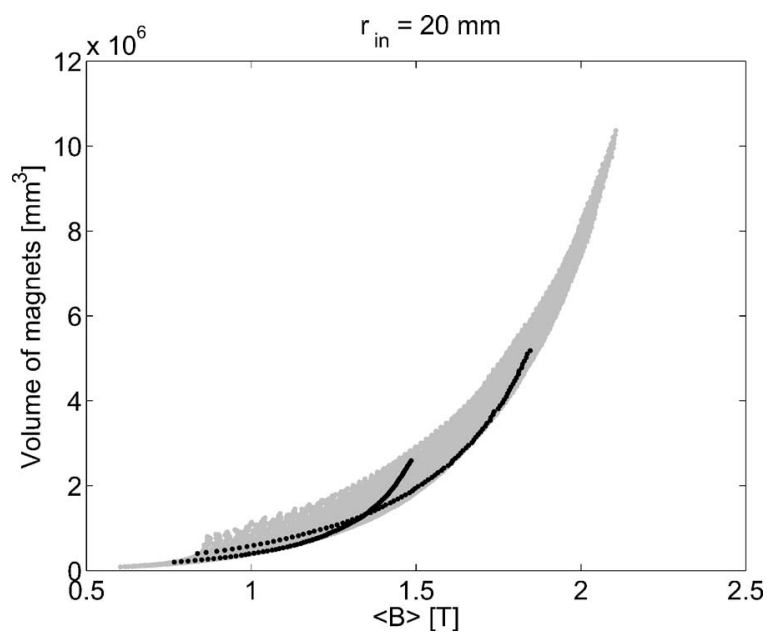

FIG. 7. The total volume of the magnetic material as a function of the mean flux density for $r_{\text {in }}=20 \mathrm{~mm}$. The data points somewhat resemble a feather, and so this plot will be referred to as the "feather plot." The data have been produced in series where $r_{\mathrm{ex}}$ has been fixed and $L$ has been varied. Two of these data series have been highlighted in black and, starting from the left, both series can be seen to first approach the rightwards edge of the feather and then leave it again. Similar plots exist for $r_{\text {in }}=10$ and $30 \mathrm{~mm}$.

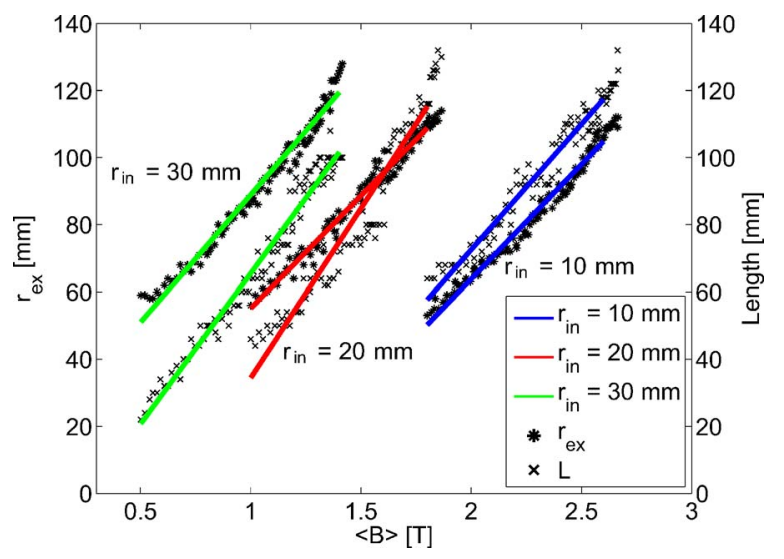

FIG. 8. (Color online) The optimal $r_{\mathrm{ex}}$ and $L$ as functions of the mean flux density in the cylinder bore for Halbach cylinders with $r_{\text {in }}=10,20$, and 30 $\mathrm{mm}$. Building a Halbach cylinder with dimensions different from the dimensions given here means that more magnetic material is used than need be, if one is only interested in obtaining the maximum flux density possible and does not care about the size of the cylinder bore.

for the Halbach cylinder with the minimum volume of magnets at a given mean flux density. Straight lines have been fitted to the data.

The conclusion of this parameter investigation is twofold. First, it can be concluded from Figs. 4-6 that it is possible, at a constant mean flux density, to increase the volume of the bore significantly by only increasing the volume of the magnets slightly for a Halbach cylinder with a fixed $r_{\text {in }}$. Second, the length and external radius of the minimum magnetic material Halbach cylinder at a given mean flux density was found and can be read off directly from Fig. 8. Thus if one wishes to build a Halbach cylinder with a given mean flux density and the limiting factor is the price of magnetic material, one should always choose the optimal configuration from this figure.

A few remarks on the precision of the simulations are in order. With the chosen boundary conditions, i.e., a magnetically insulating computational volume, it is important that the computational volume is large enough that the insulating boundaries do not effect the calculations. Also, as the solution method used is a finite element method, the mesh applied to the geometry must be as detailed as needed for the desired precision. The resolution of the mesh used for the simulations presented in this paper is chosen such that the results have a high degree of precision. To give an example, the mean flux density of the cylinder bore was calculated at different precisions for a random Halbach cylinder. The Halbach cylinder chosen had $r_{\text {in }}=20 \mathrm{~mm}, r_{\mathrm{ex}}=102 \mathrm{~mm}$, and $L$ $=70 \mathrm{~mm}$, and a mean flux density of $1.54 \mathrm{~T}$. This result, calculated using the precision used throughout this paper, deviated by only $1.13 \%$ from a simulation using $173 \%$ more mesh elements all in all, and $1845 \%$ more mesh elements in the cylinder bore. The influence of the size of the computational volume on the mean flux density in the bore has also been tested for a number of different values of $r_{\mathrm{in}}, r_{\mathrm{ex}}$, and $L$ and found to be less than $1 \%$. Thus we conclude that at least the relative precision of the numerical experiments is satisfactory.

Although the above results are useful in choosing the 

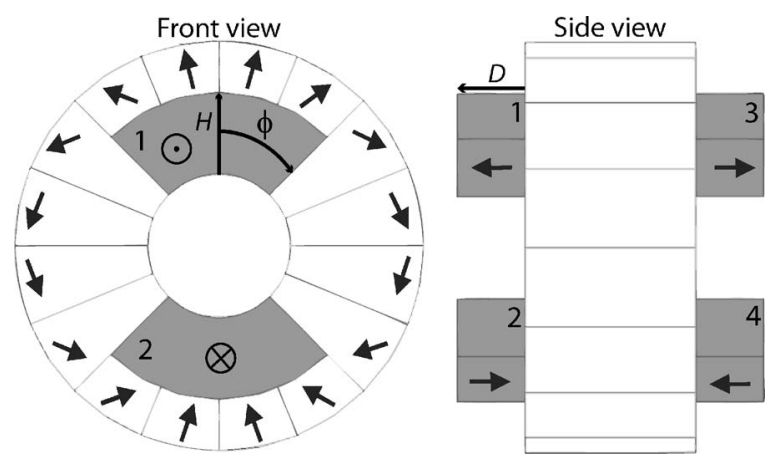

FIG. 9. This figure shows the height, $H$, angular span, $\phi$, and depth, $D$, of the additional round blocks, colored in gray. The blocks are always symmetrically placed. The black arrows show the direction of magnetization. The additional blocks diagonally opposite each other have the same direction of magnetization.

optimal Halbach cylinder design, alternative methods for improving the design of a Halbach cylinder exist. The problem with especially the short Halbach cylinders is that they lose a relatively large amount of flux through the ends of the cylinder. This is the reason that their flux density is not well described by Eq. (2). In the next section it is investigated if it is possible to limit the amount of escaping flux through the ends of the cylinder by appending blocks of permanent magnets to the end faces of the Halbach cylinder and thus in this way improve the design.

\section{IMPROVING THE HALBACH CYLINDER DESIGN}

The main loss of flux from the bore of the Halbach cylinder is through the ends of the cylinder bore. It has previously been shown ${ }^{14}$ that by "covering" the ends of the Halbach cylinder with magnetic blocks in the shape of an equipotential surface, all of the flux can be confined inside the Halbach cylinder. However, this also blocks access to the cylinder bore. Instead we propose that some of the escaping flux may be confined by placing additional magnets, of a given size and direction of magnetization, at the end faces of the cylinder, in such a way that they do not block access to the cylinder bore but still increase the flux density in the cylinder bore and ensure a low flux density outside of the Halbach cylinder.

In this section we investigate what specific design and placement are optimal for these additional magnets. We also discuss whether it is better to use the additional magnets or if one might as well use the additional magnetic material for building a larger Halbach cylinder.

To maximize the amount of magnetic material capable of being used in the additional blocks we use a design of the additional blocks that follows the curvature of the cylinder bore, i.e., a circular design as can be seen in Fig. 9. In total four additional blocks are used, placed symmetrically around the Halbach cylinder symmetry axis. In this design an additional block is characterized by three parameters, namely the angular extent of a block, $\phi$, the block's depth, $D$, and the height, $H$. The direction of magnetization of the individual additional block is perpendicular to the Halbach cylinder end face. Furthermore, the blocks diagonally opposite have the same direction of magnetization.
TABLE II. The additional magnets were placed on the four different Halbach cylinders given in column 5 . The parameters of the additional blocks were varied as given in this table, resulting in $10 \times 8 \times 8$ different configurations of the additional blocks for each Halbach cylinder.

\begin{tabular}{lrccc}
\hline \hline & From & To & Step size & Halbach dimensions $(\mathrm{mm})$ \\
\hline$\phi\left({ }^{\circ}\right)$ & 10 & 80 & 10 & $L=100$ \\
$H(\mathrm{~mm})$ & 100 & 30 & 10 & $r_{\mathrm{ex}}=100$ \\
$D(\mathrm{~mm})$ & 50 & 5 & 5 & $r_{\mathrm{in}}=20$ \\
& & & & $L=92$ \\
$\phi\left(^{\circ}\right)$ & 10 & 80 & 10 & $r_{\mathrm{ex}}=130$ \\
$H(\mathrm{~mm})$ & 130 & 25 & 15 & $r_{\mathrm{in}}=20$ \\
$D(\mathrm{~mm})$ & 46 & 4.6 & 4.6 & $L=200$ \\
$\phi\left({ }^{\circ}\right)$ & 10 & 80 & 10 & $r_{\mathrm{ex}}=60$ \\
$H(\mathrm{~mm})$ & 60 & 25 & 5 & $r_{\mathrm{in}}=20$ \\
$D(\mathrm{~mm})$ & 100 & 10 & 10 & $L=50$ \\
$\phi\left({ }^{\circ}\right)$ & 10 & 80 & 10 & $r_{\mathrm{ex}}=130$ \\
$H(\mathrm{~mm})$ & 130 & 25 & 15 & $r_{\mathrm{in}}=20$ \\
$D(\mathrm{~mm})$ & 25 & 2.5 & 2.25 & \\
\hline \hline
\end{tabular}

The height, $H$, angular span, $\phi$, and depth, $D$, of the additional blocks are varied to find the optimal configuration for several different Halbach cylinders. Calculating the flux density for each of the original $90 \times 90 \times 3$ Halbach cylinders with additional magnets is a too time consuming task, and thus the calculations were only done on a few carefully chosen Halbach cylinder designs. These are given in Table II.

The results of the simulations are shown in Fig. 10. Here the mean flux density in the bore as a function of the total volume of the magnetic material used in the simulated design is shown. The figure shows both the Halbach cylinders without any additional blocks, and the simulations of the Halbach cylinders with additional blocks.

One can see from the figure that placing additional blocks on a relatively short Halbach cylinder will improve the mean flux density in the cylinder bore significantly.

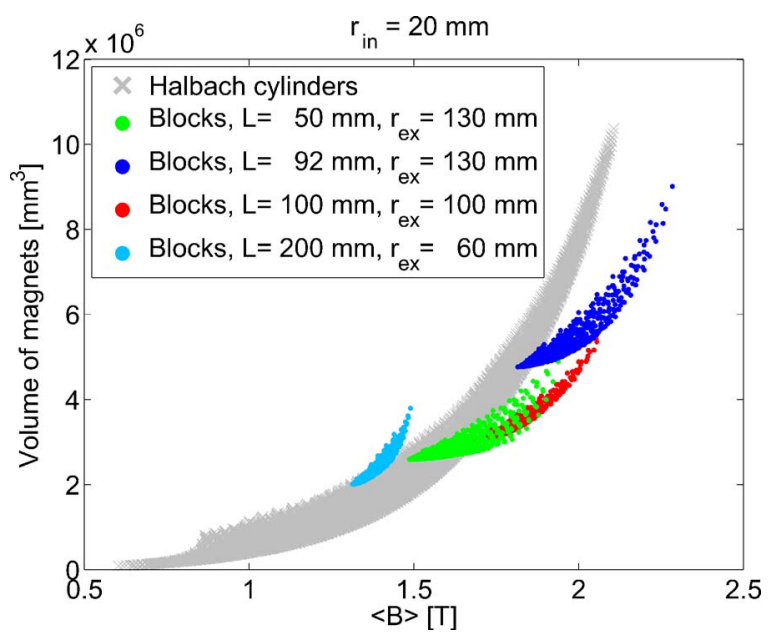

FIG. 10. (Color online) Placing additional blocks on a specific Halbach cylinder improves the mean flux density in the cylinder bore but the improvement depends on $L$ and $r_{\mathrm{ex}}$ of the Halbach cylinder. The legend shows which Halbach cylinder the additional block was placed on. Without the additional blocks the figure is identical to Fig. 7. 


\section{HALBACH CYLINDERS FOR USE IN MAGNETIC COOLING}

We have shown that using additional blocks of magnets on the sides of the Halbach cylinder can increase the mean flux density in the cylinder bore. However, in some cases the additional magnetic material might as well be used to enlarge the Halbach cylinder's external radius and in this way also increase the flux density. We will consider this more closely in the context of one particular application for Halbach cylinders, namely magnetic cooling. For this type of application the Halbach cylinder must be designed such that it has a high flux density in a large volume and with a minimum of magnetic material.

The magnetic cooling process relies on a magnetocaloric material. The temperature of such a material is increased upon the application of a magnetic field and decreased again upon the removal of the magnetic field. A large number of different materials have been suggested as the active component of a magnetic refrigeration machine. ${ }^{15}$

From experimental studies it is known that the adiabatic temperature change of gadolinium, the "benchmark" magnetocaloric material at room temperature, has a magnetocaloric effect that scales with the flux density of the magnetic field ${ }^{16}$ to the power of 0.7 . This is in good accordance with the power of $\frac{2}{3}$ predicted by mean field theory. ${ }^{17}$

However, it is not only the flux density inside the cylinder bore that is of importance to the magnetocaloric effect. The volume outside the cylinder bore where the magnetocaloric material is placed when it is moved to the "out of field" position is also important. In order to maximize the magnetocaloric effect the flux density in this region must be as low as possible. It can, of course, be argued that one could simply move the magnetocaloric material further away than right outside the end of the cylinder bore, but this would increase the physical size of the magnetic refrigeration machine. Finally, it is important that the cylinder bore has as large a volume as possible and that the volume of the magnets be as small as possible.

Taking all this into account we propose to characterize a configuration of magnets for use in magnetic cooling applications by the parameter

$$
\Lambda_{\text {cool }} \equiv\left(\left\langle B^{0.7}\right\rangle-\left\langle B_{\text {out }}^{0.7}\right\rangle\right) \frac{V_{\text {field }}}{V_{\text {mag }}} P_{\text {field }},
$$

where $V_{\text {mag }}$ is the volume of the magnets, $V_{\text {field }}$ is the volume with a high flux density, i.e., the volume of the cylinder bore, $P_{\text {field }}$ is the fraction of the total volume of the cylinder bore and the volume outside the cylinder bore that is filled with magnetocaloric material, $\left\langle B_{\text {out }}^{0.7}\right\rangle$ is the volume average of the flux density in the high flux volume, i.e., the cylinder bore, to the power of 0.7 , and $\left\langle B_{\text {out }}^{0.7}\right\rangle$ is the volume average of the flux density to the power of 0.7 in the region shown in Fig. 11, i.e., the volume just outside the cylinder bore where the magnetocaloric material is placed when it is moved out of the magnetic field. It has the same size and shape as the cylinder bore.

The magnetic cooling parameter is shown for the Halbach cylinders without additional blocks and with $r_{\text {in }}$

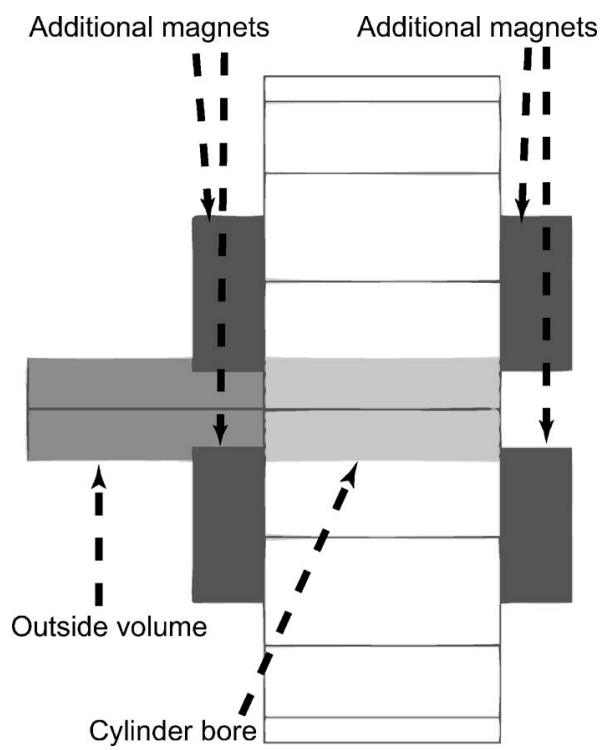

FIG. 11. A side view of the Halbach cylinder with additional blocks. The volume inside which $\left\langle B_{\text {out }}^{0.7}\right\rangle$ is calculated is shown. The volume is identical in shape to the cylinder bore, also shown, and is located directly outside the end of the cylinder bore. Also shown are the additional blocks of magnets. The edges of the 16 segments that make up the Halbach cylinder can also be seen in the figure.

$=20 \mathrm{~mm}$ in Fig. 12 for $P_{\text {field }}=0.5$, i.e., we assume that the total volume is only half filled with magnetocaloric material at any given moment. Here we see that the optimal design is the Halbach cylinder with the largest $L$ and smallest $r_{\text {ex }}$. Note that this design is not the overall optimal design, as it lies on the edge of the parameter space, i.e., simulations have not been conducted with a larger $L$ and smaller $r_{\mathrm{ex}}$.

There are several reasons that the long, thin Halbach cylinder has the largest $\Lambda_{\text {cool }}$. The primary cause is due to the fact that the loss of flux through the ends of the cylinder bore is greatly reduced in the long Halbach cylinder. Another effect is, due to its long length, the volume inside which $\left\langle B_{\text {out }}^{0.7}\right\rangle$ is calculated is also long (as previously mentioned the shape

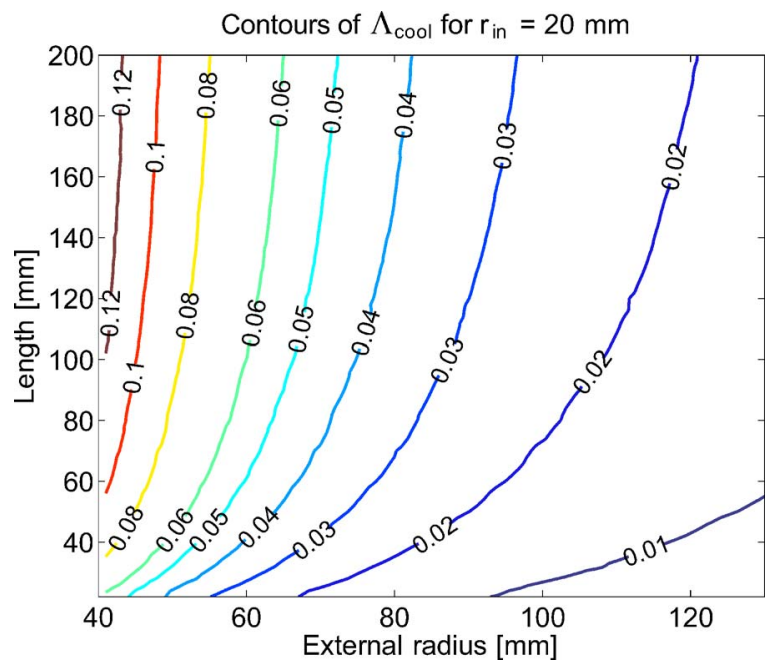

FIG. 12. (Color online) A contour plot showing the magnetic cooling parameter, $\Lambda_{\text {cool }}$, defined in Eq. (5) for the Halbach cylinders without additional blocks and with $r_{\text {in }}=20 \mathrm{~mm}$. The optimum design is the longest and thinnest Halbach cylinder. 


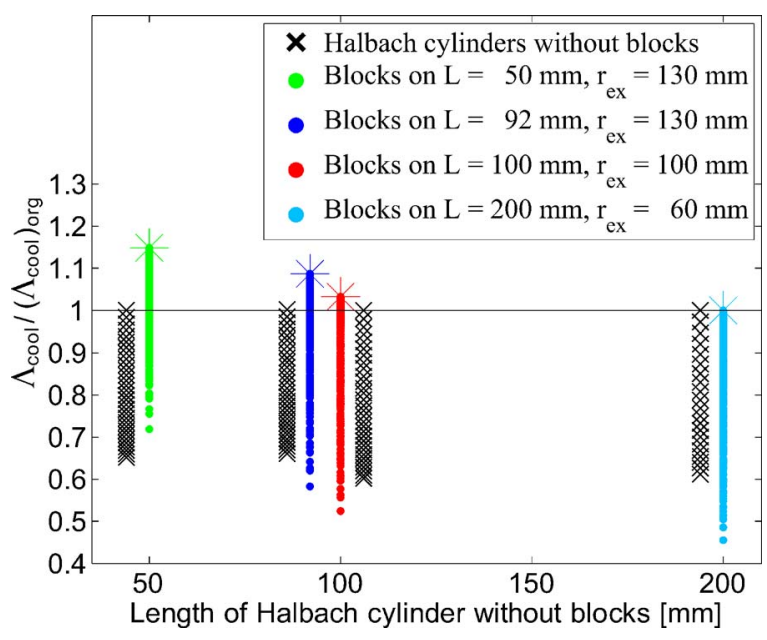

FIG. 13. (Color online) The relative improvement of the magnetic cooling parameter compared to the original Halbach cylinder without any additional blocks, for the designs listed in Table II. The design most improved is the short Halbach cylinder with a large external radius, i.e., $L=50 \mathrm{~mm}, r_{\mathrm{ex}}$ $=130 \mathrm{~mm}$. For each Halbach cylinder the best configuration of the additional blocks have been marked by a star. The black crosses in the figure are Halbach cylinders with the same $r_{\text {in }}$ and $L$ as the Halbach cylinder with blocks, but with a bigger $r_{\mathrm{ex}}$ and no blocks. The black crosses are displaced by $\pm 6 \mathrm{~mm}$ on the $x$-axis, to make the comparison with the Halbach cylinders with additional blocks possible.

of the cylinder bore and this volume are identical), and thus the end furthest from the cylinder bore will only experience a very small field, thus lowering $\left\langle B_{\text {out }}^{0.7}\right\rangle$. For practical applications one would choose to optimize $\Lambda_{\text {cool }}$ under a criterion of a minimum flux density in the cylinder bore, i.e., find the Halbach cylinder with the maximum $\Lambda_{\text {cool }}$ that at the same time has a minimum flux density of, e.g., $1 \mathrm{~T}$ in the cylinder bore.

We are also interested in knowing what effect the additional blocks of magnets have on the magnetic cooling parameter. In Fig. 10 we saw that the additional blocks increase the flux density in the cylinder bore, but this might not mean that $\Lambda_{\text {cool }}$ is increased as well, as additional magnetic material is also used.

In Fig. $13 \Lambda_{\text {cool }}$ is shown for the different Halbach cylinders with additional blocks, i.e., the ones given in Table II. Here we can see that some configurations of the additional blocks do increase $\Lambda_{\text {cool }}$ by as much as $\sim 15 \%$. Shown in the figure are also Halbach cylinders with no additional blocks that have the same $r_{\text {in }}$ and $L$ as the Halbach cylinders with additional blocks but where $r_{\text {ex }}$ has been expanded by up to $30 \mathrm{~mm}$. These are shown such that it can be estimated if it is better to spend any additional magnetic material on the additional blocks or on enlarging the external radius of the Halbach cylinder. As one can see from the figure, in three of the cases it is better to spend the additional magnetic material on the additional blocks.

The design that is the most improved by the additional blocks is a short Halbach cylinder with a large external radius, i.e., $L=50 \mathrm{~mm}, r_{\mathrm{ex}}=130 \mathrm{~mm}$. For the longest Halbach cylinder with the smallest external radius, $L=200 \mathrm{~mm}, r_{\mathrm{ex}}$ $=60 \mathrm{~mm}$, the additional blocks do not improve $\Lambda_{\text {cool }}$. The reason for this is that the short designs are also the ones that
TABLE III. The optimal configuration of the additional blocks, i.e., the configurations that maximize the magnetic cooling parameter. $\left(\Lambda_{\text {cool }}\right)_{\text {org }}$ refers to the "original" Halbach cylinder without any additional blocks.

\begin{tabular}{lcccc}
\hline \hline Halbach dimensions $(\mathrm{mm})$ & $\phi\left({ }^{\circ}\right)$ & $H(\mathrm{~mm})$ & $D(\mathrm{~mm})$ & $\Lambda_{\text {cool }} /\left(\Lambda_{\text {cool }}\right)_{\mathrm{org}}$ \\
\hline$r_{\mathrm{ex}}=130, L=50$ & 80 & 47.5 & 22.5 & 1.15 \\
$r_{\mathrm{ex}}=130, L=92$ & 60 & 61.2 & 27.6 & 1.09 \\
$r_{\mathrm{ex}}=100, L=100$ & 60 & 50 & 25 & 1.03 \\
$r_{\mathrm{ex}}=60, L=200$ & 60 & 30 & 10 & 1.00 \\
\hline \hline
\end{tabular}

have the greatest loss of flux through the ends of the cylinder bore, which is exactly what the additional magnets reduce.

The optimal design of the additional blocks, i.e., the angular span, height, and depth, varies between the four Halbach cylinders presented in Fig. 13, and the results can be read in Table III. Here it is seen that the optimal design of the additional blocks seems to be an angle span of around $60^{\circ}$, a height in the range $45-60 \mathrm{~mm}$, and a depth in the range $22.5-27.5 \mathrm{~mm}$, at least for the three systems used in these simulations. The parameters were varied as given in Table II.

It can thus be concluded that for a short Halbach cylinder with a large external radius it is possible to optimize the magnetic cooling parameter by using additional magnets placed at the ends of the cylinder. However, as can be seen by comparing Figs. 12 and 13, the improvement gained by using the additional blocks is small compared to building a long Halbach cylinder with a small $r_{\mathrm{ex}}$ in the first place. For example, the design improved the most by the additional blocks, $r_{\mathrm{ex}}=130 \mathrm{~mm}$ and $L=50 \mathrm{~mm}$, has the magnetic cooling parameter improved 1.15 times. This is not as impressive when one considers that the magnetic cooling parameter for this Halbach cylinder has a value of 0.011 (the lower right corner in Fig. 12), and even multiplied by 1.15 this is still much lower than the longer Halbach cylinders. However, in specific cases with a limited geometry due to the application the additional blocks can still be used to improve the flux density.

\section{A. Homogeneity of the field}

In most Halbach cylinder applications it is not only the flux density that is important but also the homogeneity of the field in the cylinder bore.

To characterize the homogeneity of the flux density in the cylinder bore the quantity

$$
\eta \equiv \frac{\left\langle B^{2}\right\rangle-\langle B\rangle^{2}}{\left\langle B^{2}\right\rangle}
$$

where the angled brackets denoting volume average, is defined. In Fig. 14 this parameter is shown for the Halbach cylinders with additional blocks. Also shown in the figure are Halbach cylinders with the same $r_{\text {in }}$ and $L$ as the Halbach cylinder with additional blocks but with a larger $r_{\mathrm{ex}}$ and no blocks. It can clearly be seen that the no-block designs with larger $r_{\mathrm{ex}}$ have a homogeneity parameter comparable to the "original" Halbach cylinder without blocks, while a number of the designs with additional blocks clearly improve the homogeneity of the field in the cylinder bore, i.e., lower $\eta / \eta_{\text {org }}$. 


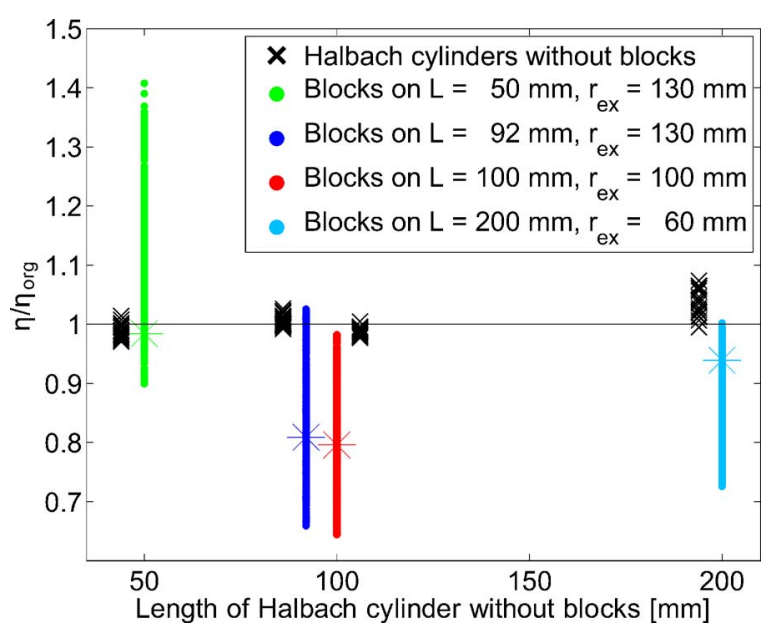

FIG. 14. (Color online) The parameter $\eta / \eta_{\text {org }}$ describing the homogeneity of the field in the Halbach cylinder bore. The data point marked with a star represents the best data point from Fig. 13. The black crosses are Halbach cylinders with the same $r_{\text {in }}$ and $L$ as the Halbach cylinder with additional blocks but with a larger $r_{\mathrm{ex}}$ and no blocks. The black crosses are displaced by $\pm 6 \mathrm{~mm}$ on the $x$-axis to make the comparison with the Halbach cylinders with additional blocks possible.

\section{B. Direction of magnetization}

As previously mentioned, the additional blocks all have a direction of magnetization perpendicular to the end face of the Halbach cylinder. This might not be the optimal configuration, so various directions of magnetization have been tested to find the greatest enhancement of the flux density. The direction of magnetization was given by $\left(0, B_{r} \cos (\theta), B_{r} \sin (\theta)\right)$ where $\theta$ was varied in steps of $1^{\circ}$. The Halbach cylinder symmetry axis is oriented along the $z$-axis.

The result indicates that the mean flux density in the bore could only be improved by less than $1 \%$ by changing the direction of magnetization from the $90^{\circ}$ orientation used in the preceding simulations.

\section{DISCUSSION}

It is important to discuss the possible influence of the coercivity of the individual magnets in the Halbach cylinder assembly. A serious problem in this context is that the assumed linearity of the magnets is only valid when the magnetic field is above the value of the intrinsic (polarization) coercivity, $H_{c}$. For typical $1.4 \mathrm{~T} \mathrm{NdFeB}$ magnets $\mu_{0} H_{c}$ is around $1.2 \mathrm{~T}$ at room temperature. Once the reverse component of the magnetic field reaches this value the linearity of the magnets breaks down and a small increase in the magnetic field will reverse the direction of magnetization of the magnet. We have not modeled this nonlinearity but have assumed that the linear relation is always valid. This is of course problematic when the magnetic field strength is too high. The reason the nonlinearity is not modeled is that due to hysteresis a complete history of the magnet material would be needed, including the physical building of the Halbach array, and this is not possible to model.

The part of the Halbach cylinder where this is a problem is around the inner equator. ${ }^{18}$ In Fig. 15 the projection of the magnetic field intensity along the direction of the remanent

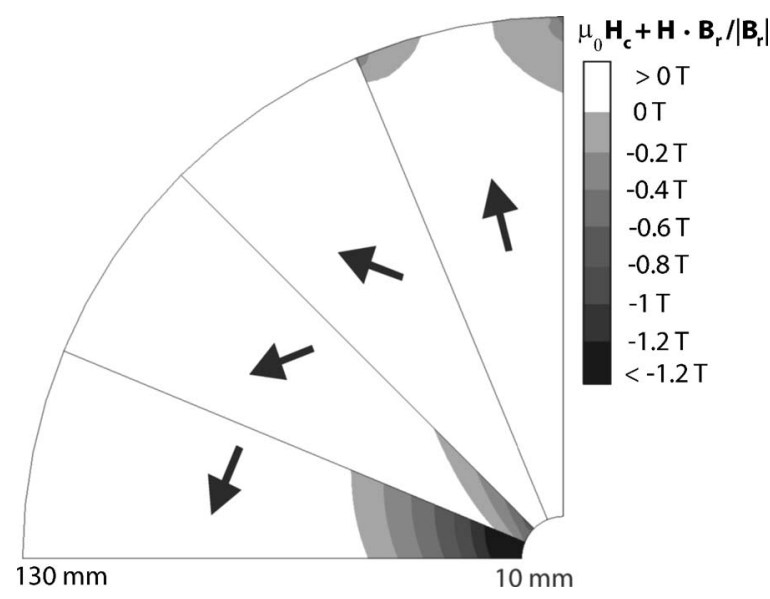

FIG. 15. A quarter of a 2D Halbach cylinder with $r_{\mathrm{in}}=10 \mathrm{~mm}$ and $r_{\mathrm{ex}}$ $=130 \mathrm{~mm}$. Shown as a color map is the expression $\mu_{\mathbf{0}} \mathbf{H}_{\mathbf{c}}+\mathbf{H} \cdot \mathbf{B}_{\mathbf{r}} /\left|\mathbf{B}_{\mathbf{r}}\right|$. When this expression is negative the magnetic field intensity is stronger than the intrinsic coercivity of the magnet (taken to be $\mu_{0} H_{c}=1.2 \mathrm{~T}$ ) and the magnet will be reversed. The directions of the magnetization of the blocks are shown as black arrows.

magnetism is shown for the largest Halbach considered in this paper. Anisotropy is typically larger than coercivity for $\mathrm{NdFeB}$ magnets so any reverse field component is important. ${ }^{18}$ It can be seen that the magnetic field is strong enough to reverse the direction of magnetization of typical industry $1.4 \mathrm{~T}$ magnets at several locations.

The problem is present for a Halbach cylinder with a flux density in the bore larger than $1.2 \mathrm{~T}$. To overcome this problem one can replace the magnets in the affected volume of the Halbach cylinder with magnets with a higher intrinsic coercivity. Such magnets are readily available at slightly lower remanences, e.g., a typical industry $\mathrm{NdFeB}$ magnet with a remanence of $1.2 \mathrm{~T}$ has $\mu_{0} H_{c}=3.2 \mathrm{~T}$, which is sufficiently strong to keep the relative permeability constant and thus the material linear. Otherwise the best solution is to remove the magnets from this part of the Halbach cylinder and replace them with a nonmagnetic material.

An additional remark on the conducted numerical simulations is that because the Halbach cylinder consists of magnets with a relative permeability close to one, the magnetostatic problem of calculating the flux density is linear in the remanence. This means that the mean flux density both inside and outside the Halbach cylinder depends linearly on the remanence of the magnets. In this paper we have used magnets with a remanence of $1.4 \mathrm{~T}$. If one would, e.g., replace all these magnets in the Halbach cylinder with magnets with a remanence of $1.2 \mathrm{~T}$, the mean flux density both inside and outside the Halbach cylinder would decrease by a factor of $1.2 / 1.4=0.86$. This has been verified numerically.

There are still factors that have not been taken into account. We have, for example, discussed the use of additional blocks while taking their shape for granted. It is necessary to test if the circular design used for the additional blocks is the proper design to use. One could just as well have used, e.g., a square design of the additional blocks. It is also important to investigate the effect of the additional blocks on a much larger sample of Halbach designs, including designs with varying internal radii. 


\section{CONCLUSION}

In this paper we found the optimal values of $r_{\mathrm{ex}}$ and $L$ for a Halbach cylinder with a given mean flux density and $r_{\text {in }}$. These configurations have the smallest volume of the magnet possible for a given mean flux density in the cylinder bore. Also, we found that placing blocks of additional permanent magnets on the sides of the Halbach cylinder can improve the flux density in the cylinder bore significantly. Finally, we introduced a magnetic cooling efficiency parameter, $\Lambda_{\text {cool }}$, and showed that the additional blocks can improve this by as much as $15 \%$ compared to ordinary Halbach cylinders. However, one must always take care that the polarization coercivity, $H_{c}$, is always higher than the flux density in the Halbach cylinder gap.

\section{ACKNOWLEDGMENTS}

The authors would like to acknowledge the support of the Programme Commission on Energy and Environment (EnMi) (Contract No. 2104-06-0032), which is part of the Danish Council for Strategic Research.

${ }^{1}$ M. Sullivan, G. Bowden, S. Ecklund, D. Jensen, M. Nordby, A. Ringwall, and Z. Wolf, Proceedings of the 1997 Particle Accelerator Conference 3,
3330 (1998).

${ }^{2}$ S. Appelt, H. Kühn, F. W. Häsing, and B. Blümich, Nat. Phys. 2, 105 (2006).

${ }^{3}$ J. M. D. Coey, J. Magn. Magn. Mater. 248, 441 (2002).

${ }^{4}$ J. C. Mallinson, IEEE Trans. Magn. 9, 678 (1973).

${ }^{5}$ K. Halbach, Nucl. Instrum. Methods 169, 1 (1980).

${ }^{6}$ T. R. Ni Mhiochain, D. Weaire, S. M. McMurry, and J. M. D. Coey, J. Appl. Phys. 86, 6412 (1999).

${ }^{7}$ X. N. Xu, D. W. Lu, G. Q. Yuan, Y. S. Han, and X. Jin, J. Appl. Phys. 95, 6302 (2004).

${ }^{8}$ G. Moresi, and R. Magin, Concepts Magn. Reson., Part B 19B(1), 35 (2003).

${ }^{9}$ COMSOL AB, Tegnérgatan 23, SE-111 40 Stockholm, Sweden.

${ }^{10}$ Comsol, Comsol Multiphysics Model Library, 3rd ed. COMSOL AB, Chalmers Teknikpark 41288 G (2005).

${ }^{11}$ O. Schenk, K. Gärtner, W. Fichtner, and A. Stricker, FGCS, Future Gener. Comput. Syst. 18, 69 (2001).

${ }^{12}$ O. Schenk and K. Gärtner, Parallel Comput. 28, 187 (2002).

${ }^{13}$ Standard Specifications for Permanent Magnet Materials, Magnetic Materials Producers Association, 8 South Michigan Avenue, Suite 1000, Chicago, IL 60603

${ }^{14}$ E. Potenziani, J. P. Clarke, and H. A. Leupold, J. Appl. Phys. 61, 3466 (1987).

${ }^{15}$ K. A. Gschneidner, Jr., V. K. Pecharsky, and A. O. Tsokol, Rep. Prog. Phys. 68, 1479 (2005).

${ }^{16}$ V. K. Pecharsky and K. A. Gschneidner, Jr., Int. J. Refrig. 29, 1239 (2006).

${ }^{17}$ H. Oesterreicher and F. T. Parker, J. Appl. Phys. 55, 4334 (1984).

${ }^{18}$ F. Bloch, O. Cugat, G. Meunier, and J. C. Toussaint, IEEE Trans. Magn. 34, 2465 (1998). 